\title{
Global stability of a modified Leslie-Gower model with Beddington-DeAngelis functional response
}

\section{Shengbin Yu*}

\section{"Correspondence:}

yushengbin.8@163.com

Sunshine College, Fuzhou

University, Fuzhou, Fujian 350015,

P.R. China

\begin{abstract}
A predator-prey model with modified Leslie-Gower and Beddington-DeAngelis functional response is studied. The local stability of the equilibria and the permanence of the system are investigated. By applying the fluctuation lemma, qualitative analysis and Lyapunov direct method, respectively, three sufficient conditions on the global asymptotic stability of a positive equilibrium are obtained.
\end{abstract} MSC: 34D23; 92D25; 34D20; 34D40

Keywords: Leslie-Gower; permanence; global asymptotic stability; Lyapunov function; Dulac function; fluctuation lemma

\section{Introduction}

As pointed out by Berryman [1], the dynamic relationship between predators and their prey has long been and will continue to be one of the dominant themes in both ecology and mathematical ecology due to its universal existence and importance. Leslie [2, 3] introduced the following famous Leslie predator-prey system:

$$
\begin{aligned}
& \dot{x}(t)=\left(r_{1}-b_{1} x(t)\right) x(t)-p(x(t)) y(t), \\
& \dot{y}(t)=\left(r_{2}-a_{2} \frac{y(t)}{x(t)}\right) y(t),
\end{aligned}
$$

where $x(t), y(t)$ stand for the population (the density) of the prey and the predator at time $t$, respectively. The parameters $r_{1}$ and $r_{2}$ are the intrinsic growth rates of the prey and the predator, respectively. $b_{1}$ measures the strength of competition among individuals of species $x$. The value $\frac{r_{1}}{b_{1}}$ is the carrying capacity of the prey in the absence of predation. The predator consumes the prey according to the functional response $p(x)$ and carries capacity $\frac{x}{a_{2}}$. The parameter $a_{2}$ is a measure of the food quantity that the prey provides converted to predator birth. The term $y / x$ is the Leslie-Gower term which measures the loss in the predator population due to rarity (per capita $y / x$ ) of its favorite food. Leslie model is a predator-prey model where the carrying capacity of the predator is proportional to the number of prey, stressing the fact that there are upper limits to the rates of increase in both prey $x$ and predator $y$, which are not recognized in the Lotka-Volterra model. These upper limits can be approached under favorable conditions: for the predators, when the number of prey per predator is large; for the prey, when the number of predators (and 
perhaps the number of prey also) is small [4]. For more details of the model, one can see [4-9] and the references cited therein. Holling [10] suggested three different kinds of functional response for different kinds of species to model the phenomena of predation, which made the standard Lotka-Volterra system more realistic. When $p(x)=\frac{a_{1} x}{x+k_{1}}$, the functional response $p(x)$ is called Holling-type II.

Recently, Aziz-Alaoui and Daher Okiye [11] pointed out that in the case of severe scarcity, $y$ can switch over to other populations but its growth will be limited by the fact that its most favorite food $x$ is not available in abundance. To solve such a problem, they suggested to add a positive constant $d$ to the denominator and proposed the following predator-prey model with modified Leslie-Gower and Holling-type II schemes:

$$
\begin{aligned}
& \dot{x}(t)=\left(r_{1}-b_{1} x(t)-\frac{a_{1} y(t)}{x(t)+k_{1}}\right) x(t), \\
& \dot{y}(t)=\left(r_{2}-\frac{a_{2} y(t)}{x(t)+k_{2}}\right) y(t),
\end{aligned}
$$

where $r_{1}, b_{1}, r_{2}, a_{2}$ have the same meaning as in models (1.1). $a_{1}$ is the maximum value of the per capita reduction rate of $x$ due to $y, k_{1}$ (respectively, $k_{2}$ ) measures the extent to which the environment provides protection to prey $x$ (respectively, to the predator $y$ ). The authors studied the boundedness and global stability of positive equilibrium of system (1.2). Since then, system (1.2) and its non-autonomous versions have been studied by incorporating delay and impulses, harvesting and so on (see, for example, [12-29]). In [12], we studied the structure, linearized stability and the global asymptotic stability of equilibria of (1.2). Nindjin et al. [13] incorporated time delay to system (1.2) and studied the global stability and persistence of the delayed system by using the Lyapunov functional. Yafia et al. [14] and [15] further studied the occurrence of Hopf bifurcation at equilibrium by using the time delay as a parameter of bifurcation. Nindjin and Aziz-Alaoui [16] studied uniform persistence and global stability of three Leslie-Gower-type species food chain system. Gakkhar and Singh [17] studied the dynamic behaviors of a modified Leslie-Gower predator-prey system with seasonally varying parameters. Guo and Song [18], Song and $\mathrm{Li}$ [19] further considered the influence of impulsive effect. Zhu and Wang [20] obtained sufficient conditions for the existence and global attractivity of positive periodic solutions of system (1.2) with periodic coefficients. Liu and Wang [21] considered a stochastic predator-prey system with modified Leslie-Gower and Holling-type II schemes with Lévy jumps. The results showed that the Lévy jumps can change the properties of the population systems significantly. Kar and Ghorai [22] obtained local stability, global stability, influence of harvesting and bifurcation of a delayed predator-prey system in the presence of harvesting. Two stage-structured predator-prey models with modified LeslieGower and Holling-type II schemes were studied in [23-25]. Gupta and Chandra [26] discussed the permanence, stability and bifurcation (saddle-node bifurcation, transcritical, Hopf-Andronov and Bogdanov-Takens) of a modified Leslie-Gower prey-predator model with Michaelis-Menten type prey harvesting. Ji et al. $[27,28]$ showed there was a stationary distribution of a predator-prey model with modified Leslie-Gower and Hollingtype II schemes with stochastic perturbation and it has ergodic property. Lian and Xu [29] discussed the Hopf bifurcation of a predator-prey system with Holling type IV functional response and time delay. 
As we all know, the functional response can be classified into two types: prey-dependent and predator-dependent. Prey-dependent depends on prey density only, while predatordependent means that the functional response is a function of both the preys and the predators densities. Recently, the prey-dependent functional responses have been challenged by several ecologists. There is a growing explicit biological and physiological evidence [30-32] that in many situations, especially when the predator has to search for food (and therefore has to shave or compete for food), a more suitable general predator-prey theory should be predator-dependent. This is supported by numerous fields and laboratory experiments and observations [33, 34]. Starting from this argument and the traditional prey-dependent-only model, Arditi and Ginzburg [33] first proposed the ratiodependent predator-prey model. Many authors have observed that the ratio-dependent models can exhibit much richer, more complicated and more reasonable or acceptable dynamics, but it has somewhat singular behavior at low densities which has been the source of controversy [35]. For the ratio-dependent predator-prey models, one can refer to [3639].

Beddington-DeAngelis functional response $\frac{\alpha x}{a+b x+c y}$ was first proposed by Beddington and DeAngelis [40, 41]. Predator-prey model with Beddington-DeAngelis functional response has rich dynamical features, which can describe the species and the ecological systems more reasonably. Beddington-DeAngelis functional response is similar to the wellknown Holling type II functional response but has an extra term $c y$ in the denominator modeling mutual interference among predators and has some of the same qualitative features as the ratio-dependent form but avoids some of the singular behaviors of ratiodependent models at low densities.

On the other hand, in 2001, Skalski and Gilliam [42] have presented statistical evidence from nineteen predator-prey systems that three predator-dependent functional responses (Beddington-DeAngelis, Crowley-Martin, and Hassell-Varley) can provide better description of predator feeding over a range of predator-prey abundances. In some cases, the Beddington-DeAngelis type preformed even better. Theoretical studies have shown that the dynamics of models with predator-dependent functional responses can differ considerably from those with prey-dependent functional responses. Although much progress has been seen in the study of predator-prey models with modified Leslie-Gower (see [11-29]), to the best of the authors' knowledge, seldom did scholars consider the modified LeslieGower model with Beddington-DeAngelis functional response. Stimulated by above reasons, in this paper we will incorporate the Beddington-DeAngelis functional response into model (1.2) and consider the following model which is the generalization of model (1.2):

$$
\begin{aligned}
& \dot{x}(t)=\left(r_{1}-p x(t)-\frac{\alpha y(t)}{a+b x(t)+c y(t)}\right) x(t), \\
& \dot{y}(t)=\left(r_{2}-\frac{\beta y(t)}{x(t)+k}\right) y(t),
\end{aligned}
$$

with initial conditions $x(0)>0$ and $y(0)>0$. The parameters $r_{1}, p, \alpha, a, b, c, r_{2}, \beta$ and $k$ are positive constants and have the same meaning as in model (1.2).

It is easy to see that both the first quadrant $R_{+}^{2}$ and the positive first quadrant Int $R_{+}^{2}$ are invariant for system (1.3). As a result, solutions $(x(t), y(t))$ to (1.3) with $(x(0), y(0)) \in \operatorname{Int} R_{+}^{2}$ are all positive solutions. 
The rest of this paper is organized as follows. In Section 2, we discuss the structure of nonnegative equilibria to (1.3) and their local stability, which motivates us to study permanence and global stability of (1.3) respectively in Section 3 and Section 4.

For more works on this direction, one could refer to [43-51] and the references cited therein.

\section{Nonnegative equilibria and their local stability}

The Jacobian matrix of system (1.3) is

$$
J=\left(\begin{array}{cc}
r_{1}-2 p x-\frac{\alpha y(a+c y)}{(a+b x+c y)^{2}} & -\frac{\alpha x(a+b x)}{(a+b x+c y)^{2}} \\
\frac{\beta y^{2}}{(x+k)^{2}} & r_{2}-\frac{2 \beta y}{x+k}
\end{array}\right) .
$$

An equilibrium $E$ of (1.3) is (linearly) stable if the real parts of both eigenvalues of $J(E)$ are negative, and therefore a sufficient condition for stability is

$$
\operatorname{tr}(J(E))<0 \quad \text { and } \quad \operatorname{det}(J(E))>0 .
$$

Obviously, (1.3) has three boundary equilibria, $E_{0}=(0,0), E_{1}=\left(\frac{r_{1}}{p}, 0\right)$ and $E_{2}=\left(0, \frac{r_{2} k}{\beta}\right)$, whose Jacobian matrices are

$$
\begin{aligned}
& J\left(E_{0}\right)=\left(\begin{array}{cc}
r_{1} & 0 \\
0 & r_{2}
\end{array}\right), \\
& J\left(E_{1}\right)=\left(\begin{array}{cc}
-r_{1} & -\frac{\alpha r_{1}}{a p+b r_{1}} \\
0 & r_{2}
\end{array}\right),
\end{aligned}
$$

and

$$
J\left(E_{2}\right)=\left(\begin{array}{cc}
r_{1}-\frac{\alpha r_{2} k}{a \beta+c r_{2} k} & 0 \\
\frac{r_{2}^{2}}{\beta} & -r_{2}
\end{array}\right),
$$

respectively. As a direct consequence of (2.1), we have the following result.

\section{Proposition 2.1}

(i) Both $E_{0}$ and $E_{1}$ are unstable.

(ii) $E_{2}$ is locally asymptotically stable if $\alpha r_{2} k>a r_{1} \beta+r_{1} r_{2} c k$, while it is unstable if $\alpha r_{2} k<a r_{1} \beta+r_{1} r_{2} c k$.

Besides the three boundary equilibria, (1.3) may have (componentwise) positive equilibria. Suppose that $\hat{E}=(\hat{x}, \hat{y})$ is such an equilibrium. Then

$$
\begin{aligned}
& r_{1}-p \hat{x}=\frac{\alpha \hat{y}}{a+b \hat{x}+c \hat{y}}, \\
& \hat{y}=\frac{r_{2}(\hat{x}+k)}{\beta} .
\end{aligned}
$$


One can easily see that $\hat{x}$ satisfies

$$
\left(r_{2} c p+b p \beta\right) \hat{x}^{2}+B \hat{x}+\alpha r_{2} k-a r_{1} \beta-r_{1} r_{2} c k=0,
$$

where $B \triangleq \alpha r_{2}+p r_{2} c k+a p \beta-r_{1} r_{2} c-b r_{1} \beta$. Moreover, for convenience, we denote

$$
\Delta \triangleq B^{2}-4\left(r_{2} c p+b p \beta\right)\left(\alpha r_{2} k-a r_{1} \beta-r_{1} r_{2} c k\right)
$$

Hence, we have the following result.

Proposition 2.2 Suppose that

$\left(\mathrm{H}_{0}\right) \quad \alpha r_{2} k<a r_{1} \beta+r_{1} r_{2} c k$

holds, then system (1.3) has a unique positive equilibrium $\hat{E}=(\hat{x}, \hat{y})$, where

$$
\hat{x}=\frac{-B+\sqrt{\Delta}}{2\left(r_{2} c p+b p \beta\right)} \quad \text { and } \quad \hat{y}=\frac{r_{2}(\hat{x}+k)}{\beta} \text {. }
$$

We now want to study the stability of the positive equilibrium $\hat{E}=(\hat{x}, \hat{y})$. It follows from the Jacobian matrix of systems (1.3) and (2.2) that

$$
\begin{aligned}
\operatorname{tr}(J(\hat{E})) & =\frac{\left(r_{1} b-a p\right) \hat{x}-2 p b \hat{x}^{2}-p c \hat{x} \hat{y}}{a+b \hat{x}+c \hat{y}}-r_{2} \\
& =\frac{-\left(2 p b \beta+p c r_{2}\right) \hat{x}^{2}+\left(r_{1} b \beta-a p \beta-r_{2} b \beta-r_{2}^{2} c\right) \hat{x}-r_{2}^{2} c k-p c r_{2} k-a r_{2} \beta}{a \beta+\left(b \beta+c r_{2}\right) \hat{x}+c r_{2} k},
\end{aligned}
$$

and

$$
\begin{aligned}
\operatorname{det}(J(\hat{E}))= & \frac{r_{2}\left(2 p b \hat{x}^{2}-\left(r_{1} b-a p\right) \hat{x}+p c \hat{x} \hat{y}\right)}{a+b \hat{x}+c \hat{y}}+\frac{r_{2}^{2} \hat{x}(a+b \hat{x})\left(r_{1}-p \hat{x}\right)}{\beta(a+b \hat{x}+c \hat{y}) \hat{y}} \\
= & \frac{r_{2} \hat{x}}{a+b \hat{x}+c \hat{y}}\left(2 p b \hat{x}-\left(r_{1} b-a p\right)+\frac{p c r_{2}(\hat{x}+k)}{\beta}+\frac{(a+b \hat{x})\left(r_{1}-p \hat{x}\right)}{(\hat{x}+k)}\right) \\
= & \frac{r_{2} \hat{x}}{\beta(a+b \hat{x}+c \hat{y})(\hat{x}+k)}\left(\left(p b \beta+p c r_{2}\right) \hat{x}^{2}+2\left(p c r_{2} k+p b k \beta\right) \hat{x}\right. \\
& \left.+p c r_{2} k^{2}+a r_{1} \beta+a p k \beta-r_{1} b k \beta\right) .
\end{aligned}
$$

Thus, if $r_{1} b<a p$, we have

$$
\operatorname{tr}(J(\hat{E}))<0 \quad \text { and } \quad \operatorname{det}(J(\hat{E}))>0
$$

Hence, the following proposition follows from (2.1).

Proposition 2.3 Assume that

$$
\left(\mathrm{H}_{1}\right) \quad r_{1} b<a p
$$

holds, then the positive equilibrium $\hat{E}$ is locally asymptotically stable. 
Proposition 2.1 and Proposition 2.3 naturally motivate us to seek sufficient conditions on the global stability of $E_{2}$ and the unique positive equilibrium to (1.3). To achieve it, we need the bounds for positive solutions.

\section{Boundedness and permanence}

The following result can be proved by slightly modifying the proof of Lemma 3.2 of Chen [52] and it will play an important role in finding the bounds for positive solutions to (1.3).

Lemma 3.1 If $a>0, b>0$ and $\dot{x} \geq x(b-a x)$, when $t \geq t_{0}$ and $x\left(t_{0}\right)>0$, we have

$$
\liminf _{t \rightarrow+\infty} x(t) \geq \frac{b}{a} .
$$

If $a>0, b>0$ and $\dot{x} \leq x(b-a x)$, when $t \geq t_{0}$ and $x\left(t_{0}\right)>0$, we have

$$
\limsup _{t \rightarrow+\infty} x(t) \leq \frac{b}{a}
$$

Proposition 3.1 Let $(x(t), y(t))$ be any positive solution of $(1.3)$. Then

$$
\limsup _{t \rightarrow+\infty} x(t) \leq \frac{r_{1}}{p} \triangleq M_{1}
$$

and

$$
m_{2} \leq \liminf _{t \rightarrow+\infty} y(t) \leq \limsup _{t \rightarrow+\infty} y(t) \leq M_{2}
$$

where $m_{2} \triangleq \frac{r_{2} k}{\beta}$ and $M_{2} \triangleq \frac{r_{1} r_{2}+p r_{2} k}{p \beta}$.

Proof Since $(x(t), y(t))$ is a positive solution of (1.3), we have

$$
\begin{aligned}
& \dot{x} \leq\left(r_{1}-p x\right) x, \\
& \dot{y} \geq\left(r_{2}-\frac{\beta y}{k}\right) y .
\end{aligned}
$$

Then (3.1) and $\liminf \operatorname{in}_{t \rightarrow+\infty} y(t) \geq m_{2}$ follow directly from Lemma 3.1. Thus, for any $\varepsilon>0$, there exists $T>0$ such that

$$
x(t) \leq \frac{r_{1}}{p}+\varepsilon \quad \text { for } t \geq T .
$$

This combined with the second equation of (1.3) leads to

$$
\dot{y} \leq\left(r_{2}-\frac{\beta y}{\frac{r_{1}}{p}+\varepsilon+k}\right) y \quad \text { for } t \geq T .
$$

Using Lemma 3.1 again, one has

$$
\limsup _{t \rightarrow+\infty} y(t) \leq \frac{r_{2}\left(\frac{r_{1}}{p}+\varepsilon+k\right)}{\beta} .
$$

Thus $\lim \sup _{t \rightarrow \infty} y(t) \leq M_{2}$ by letting $\varepsilon \rightarrow 0$. 
Proposition 3.2 Suppose that

$\left(\mathrm{H}_{2}\right) \quad a r_{1} p \beta>\alpha\left(r_{1} r_{2}+p r_{2} k\right)$.

If $(x(t), y(t))$ is a positive solution to system (1.3), then

$$
\liminf _{t \rightarrow+\infty} x(t) \geq \frac{a r_{1} p \beta-\alpha\left(r_{1} r_{2}+p r_{2} k\right)}{a p^{2} \beta} \triangleq m_{1} .
$$

Proof Denote

$$
\varepsilon_{0}=\frac{a r_{1} p \beta-\alpha\left(r_{1} r_{2}+p r_{2} k\right)}{\alpha p \beta} .
$$

Then, for $\varepsilon \in\left(0, \varepsilon_{0}\right)$, we have

$$
a r_{1} p \beta>\alpha\left(r_{1} r_{2}+p r_{2} k+p \beta \varepsilon\right) .
$$

According to Proposition 3.1, there exists $T>0$ such that

$$
y(t) \leq \frac{r_{1} r_{2}+p r_{2} k}{p \beta}+\varepsilon \quad \text { for } t \geq T .
$$

This, combined with the first equation of (1.3), produces

$$
\dot{x} \geq\left(r_{1}-p x-\frac{\alpha\left(r_{1} r_{2}+p r_{2} k+p \beta \varepsilon\right)}{a p \beta}\right) x \quad \text { for } t \geq T .
$$

It follows from Lemma 3.1 that

$$
\liminf _{t \rightarrow+\infty} x(t) \geq \frac{r_{1}-\frac{\alpha\left(r_{1} r_{2}+p r_{2} k+p \beta \varepsilon\right)}{a p \beta}}{p} .
$$

This gives $\liminf _{t \rightarrow+\infty} x(t) \geq m_{1}$ by letting $\varepsilon \rightarrow 0$.

Combing Proposition 3.1 with Proposition 3.2 gives the permanence of (1.3).

Theorem 3.1 Suppose that $\left(\mathrm{H}_{2}\right)$ holds, then (1.3) is permanent.

\section{Global asymptotic stability}

The goal of this section is to establish sufficient conditions on the global asymptotic stability of equilibrium to (1.3). The first two results are proved by employing the fluctuation lemma, which is cited below for the convenience of the readers. See Hirsch et al. [53] or Tineo [54] for more details on the fluctuation lemma.

Lemma 4.1 (Fluctuation lemma) Let $x(t)$ be a bounded differentiable function on $[\alpha, \infty)$.

Then there exist sequences $\tau_{n} \rightarrow \infty$ and $\sigma_{n} \rightarrow \infty$ such that

(i) $\dot{x}\left(\tau_{n}\right) \rightarrow 0$ and $x\left(\tau_{n}\right) \rightarrow \limsup _{t \rightarrow \infty} x(t)=\bar{x}$ as $n \rightarrow \infty$;

(ii) $\dot{x}\left(\sigma_{n}\right) \rightarrow 0$ and $x\left(\sigma_{n}\right) \rightarrow \liminf _{t \rightarrow \infty} x(t)=\underline{x}$ as $n \rightarrow \infty$. 
Theorem 4.1 Assume that

$\left(\mathrm{H}_{3}\right) \quad \alpha r_{1} p \beta+b r_{1}^{2} \beta+c r_{1} r_{2} k p<\alpha p r_{2} k$.

Then $E_{2}=\left(0, \frac{r_{2} k}{\beta}\right)$ is globally asymptotically stable for system (1.3).

Proof Let $(x(t), y(t))$ be any positive solution of (1.3). According to $\left(\mathrm{H}_{3}\right)$, we can choose $\varepsilon \in\left(0, \frac{r_{2} k}{\beta}\right)$ such that

$$
\alpha r_{1} p \beta+b r_{1}^{2} \beta+c r_{1} r_{2} k p<\alpha p r_{2} k+\left(c r_{1}-b r_{1}-\alpha\right) p \beta \varepsilon .
$$

It follows from Proposition 3.1 that there exists $T>0$ such that

$$
x(t) \leq \frac{r_{1}}{p}+\varepsilon \quad \text { and } \quad y(t) \geq \frac{r_{2} k}{\beta}-\varepsilon \quad \text { for } t \geq T .
$$

These inequalities combined with the first equation of (1.3) give us

$$
\dot{x} \leq\left(r_{1}-\frac{\alpha p\left(r_{2} k-\beta \varepsilon\right)}{a p \beta+b r_{1} \beta+c r_{2} k p+(b-c) p \beta \varepsilon}\right) \quad \text { for } t \geq T,
$$

or

$$
\frac{\dot{x}}{x} \leq\left(r_{1}-\frac{\alpha p\left(r_{2} k-\beta \varepsilon\right)}{a p \beta+b r_{1} \beta+c r_{2} k p+(b-c) p \beta \varepsilon}\right) \quad \text { for } t \geq T .
$$

Integrating both sides of (4.2) on interval $[T, t]$ leads to

$$
x(t) \leq x(T) \exp \left\{\left(r_{1}-\frac{\alpha p\left(r_{2} k-\beta \varepsilon\right)}{a p \beta+b r_{1} \beta+c r_{2} k p+(b-c) p \beta \varepsilon}\right)(t-T)\right\} .
$$

Due to (4.1), one has $\lim \sup _{t \rightarrow+\infty} x(t) \leq 0$. Hence

$$
\lim _{t \rightarrow+\infty} x(t)=0 .
$$

Proposition 3.1 again tells us that $y(t)$ is bounded and $\bar{y} \triangleq \limsup _{t \rightarrow+\infty} y(t) \geq \underline{y} \triangleq$ $\liminf _{t \rightarrow+\infty} y(t)>0$. By Lemma 4.1, there exist sequences $\tau_{n} \rightarrow \infty, \sigma_{n} \rightarrow \infty$ such that $\dot{y}\left(\tau_{n}\right) \rightarrow 0, \dot{y}\left(\sigma_{n}\right) \rightarrow 0, y\left(\tau_{n}\right) \rightarrow \bar{y}$, and $y\left(\sigma_{n}\right) \rightarrow y$, as $n \rightarrow \infty$.

It follows from the second equation of (1.3) and $\lim _{t \rightarrow+\infty} x(t)=0$ that

$$
0=\left(r_{2}-\frac{\beta \bar{y}}{k}\right) \bar{y} \quad \text { or } \quad \bar{y}=\frac{r_{2} k}{\beta} .
$$

Similarly, one can show that

$$
\underline{y}=\frac{r_{2} k}{\beta} .
$$

Equation (4.4) combined with (4.5) implies that

$$
\lim _{t \rightarrow+\infty} y(t)=\frac{r_{2} k}{\beta} .
$$

It follows from (4.3) and (4.6) that $E_{2}=\left(0, \frac{r_{2} k}{\beta}\right)$ is globally asymptotically stable. 
Theorem 4.2 In addition to $\left(\mathrm{H}_{2}\right)$, further suppose that

$$
\left(\mathrm{H}_{4}\right) \quad r_{1} b \beta+\alpha r_{2} \leq 2 b p \beta m_{1}+a p \beta+c p r_{2} k+c r_{1} r_{2}
$$

where $m_{1}$ is defined in Proposition 3.2. Then system (1.3) has a unique positive equilibrium which is globally asymptotically stable.

Proof Note that $\left(\mathrm{H}_{2}\right)$ implies $\left(\mathrm{H}_{0}\right)$, thus $(1.3)$ has a unique positive equilibrium according to Proposition 2.2. Let $(x(t), y(t))$ be any positive solution of (1.3). By the results in Section $3, \bar{x} \triangleq \limsup _{t \rightarrow \infty} x(t) \geq \underline{x} \triangleq \liminf _{t \rightarrow \infty} x(t) \geq m_{1}(>0)$ and $\bar{y} \triangleq \limsup _{t \rightarrow \infty} y(t) \geq \underline{y} \triangleq$ $\liminf _{t \rightarrow \infty} y(t)>0$.

We claim that $\bar{x}=\underline{x}$. Suppose that $\bar{x}>\underline{x}$. According to Lemma 4.1, there exist sequences $\xi_{n} \rightarrow \infty, \eta_{n} \rightarrow \infty, \tau_{n} \rightarrow \infty$ and $\sigma_{n} \rightarrow \infty$, such that $\dot{x}\left(\xi_{n}\right) \rightarrow 0, \dot{x}\left(\eta_{n}\right) \rightarrow 0, x\left(\xi_{n}\right) \rightarrow \bar{x}$ and $x\left(\eta_{n}\right) \rightarrow \underline{x}, \dot{y}\left(\tau_{n}\right) \rightarrow 0, \dot{y}\left(\sigma_{n}\right) \rightarrow 0, y\left(\tau_{n}\right) \rightarrow \bar{y}$ and $y\left(\sigma_{n}\right) \rightarrow \underline{y}$, as $n \rightarrow \infty$. First, it follows from the second equation of (1.3) that

$$
\dot{y}\left(\tau_{n}\right) \leq\left(r_{2}-\frac{\beta y\left(\tau_{n}\right)}{\sup _{t \geq \tau_{n}} x\left(\tau_{n}\right)+k}\right) y\left(\tau_{n}\right)
$$

and

$$
\dot{y}\left(\tau_{n}\right) \geq\left(r_{2}-\frac{\beta y\left(\tau_{n}\right)}{\inf _{t \geq \tau_{n}} x\left(\tau_{n}\right)+k}\right) y\left(\tau_{n}\right) .
$$

Letting $n \rightarrow \infty$ gives us

$$
0 \leq\left(r_{2}-\frac{\beta \bar{y}}{\bar{x}+k}\right) \bar{y}
$$

and

$$
0 \geq\left(r_{2}-\frac{\beta \bar{y}}{\underline{x}+k}\right) \bar{y}
$$

Hence

$$
\frac{r_{2}(\underline{x}+k)}{\beta} \leq \bar{y} \leq \frac{r_{2}(\bar{x}+k)}{\beta} .
$$

Similar arguments as above also produce

$$
\frac{r_{2}(\underline{x}+k)}{\beta} \leq \underline{y} \leq \frac{r_{2}(\bar{x}+k)}{\beta} .
$$

Second, from the first equation of (1.3), we have

$$
\dot{x}\left(\xi_{n}\right)=\left(r_{1}-p x\left(\xi_{n}\right)-\frac{\alpha y\left(\xi_{n}\right)}{a+b x\left(\xi_{n}\right)+c y\left(\xi_{n}\right)}\right) x\left(\xi_{n}\right) .
$$

Equation (4.9) implies

$$
\dot{x}\left(\xi_{n}\right) \leq\left(r_{1}-p x\left(\xi_{n}\right)-\frac{\alpha \inf _{t \geq \xi_{n}} y\left(\xi_{n}\right)}{a+b x\left(\xi_{n}\right)+c \inf _{t \geq \xi_{n}} y\left(\xi_{n}\right)}\right) x\left(\xi_{n}\right) .
$$


Taking limit as $n \rightarrow \infty$, one obtains

$$
0 \leq\left(r_{1}-p \bar{x}-\frac{\alpha \underline{y}}{a+b \bar{x}+c \underline{y}}\right) \bar{x} .
$$

This, combined with (4.8), gives us

$$
0 \leq\left(r_{1}-p \bar{x}-\frac{\alpha r_{2}(\underline{x}+k)}{a \beta+b \beta \bar{x}+c r_{2}(\underline{x}+k)}\right) \bar{x} .
$$

It follows that

$$
\left(r_{1} b \beta-a p \beta\right) \bar{x}-b p \beta \bar{x}^{2}-c p r_{2} \bar{x}(\underline{x}+k)+c r_{1} r_{2} \underline{x}+a r_{1} \beta+c r_{1} r_{2} k \geq \alpha r_{2}(\underline{x}+k) .
$$

Similarly, one can show that

$$
\left(r_{1} b \beta-a p \beta\right) \underline{x}-b p \beta \underline{x}^{2}-c p r_{2} \underline{x}(\bar{x}+k)+c r_{1} r_{2} \bar{x}+a r_{1} \beta+c r_{1} r_{2} k \leq \alpha r_{2}(\bar{x}+k) .
$$

Multiplying (4.10) by -1 and adding it to (4.11), we have

$$
b p \beta\left(\bar{x}^{2}-\underline{x}^{2}\right)+\left(a p \beta-r_{1} b \beta+c p r_{2} k+c r_{1} r_{2}-\alpha r_{2}\right)(\bar{x}-\underline{x}) \leq 0 .
$$

Due to $\bar{x}>\underline{x}$, one gets

$$
b p \beta(\bar{x}+\underline{x})+\left(a p \beta+c p r_{2} k+c r_{1} r_{2}-r_{1} b \beta-\alpha r_{2}\right) \leq 0 .
$$

On the other hand,

$$
\begin{aligned}
& b p \beta(\bar{x}+\underline{x})+\left(a p \beta+c p r_{2} k+c r_{1} r_{2}-r_{1} b \beta-\alpha r_{2}\right) \\
& >2 b p \beta m_{1}+\left(a p \beta+c p r_{2} k+c r_{1} r_{2}-r_{1} b \beta-\alpha r_{2}\right) \\
& \quad \geq 0 \text { by }\left(\mathrm{H}_{4}\right),
\end{aligned}
$$

which contradicts with (4.12). Therefore, $\bar{x}=\underline{x}$ and the claim is proved. The claim implies that $\lim _{t \rightarrow+\infty} x(t)$ exists and we denote it by $x^{*}$. Then it follows from (4.7) and (4.8) that $\lim _{t \rightarrow+\infty} y(t)$ exists and $\lim _{t \rightarrow+\infty} y(t) \triangleq y^{*}=\frac{r_{2}\left(x^{*}+k\right)}{\beta}>0$. Letting $n \rightarrow \infty$ in (4.9) gives us

$$
\left(r_{2} c \beta+b p \beta\right) x^{* 2}+\left(\alpha r_{2}+p r_{2} c k+a p \beta-r_{1} r_{2} c-b r_{1} \beta\right) x^{*}+\alpha r_{2} k-a r_{1} \beta-r_{1} r_{2} c k=0 .
$$

Thus $\left(x^{*}, y^{*}\right)$ satisfies

$$
\begin{aligned}
& r_{1}-p x^{*}=\frac{\alpha y^{*}}{a+b x^{*}+c y^{*}}, \\
& y^{*}=\frac{r_{2}\left(x^{*}+k\right)}{\beta} .
\end{aligned}
$$

That is, $\left(x^{*}, y^{*}\right)$ is a positive equilibrium of (1.3). This completes the proof as the positive equilibrium is unique and so the unique equilibrium point is globally asymptotically stable. 
The following results in this section are proved by qualitative method and applying the Lyapunov direct method with the Lyapunov function.

Theorem 4.3 Assume that $\left(\mathrm{H}_{0}\right)$ and $\left(\mathrm{H}_{1}\right)$ hold, then (1.3) has a unique positive equilibrium which is globally asymptotically stable.

Proof According to Proposition 2.2, (1.3) has a unique positive equilibrium $\hat{E}=(\hat{x}, \hat{y})$. Taking Dulac function $D(x, y)=x^{-1}(a+b x+c y) y^{-2}$, we obtain

$$
\frac{\partial(D P)}{\partial x}+\frac{\partial(D Q)}{\partial y}=\frac{\left(r_{1} b-a p\right)-2 p b x-p c y}{y^{2}}-\frac{r_{2}(a+b x)}{x y^{2}}-\frac{\beta c}{x(x+k)}
$$

where $(P, Q)$ is the vector field of (1.3). By the positivity of $x, y$, it is easy to obtain that $\frac{\partial(D P)}{\partial x}+\frac{\partial(D Q)}{\partial y}<0$ if $\left(\mathrm{H}_{1}\right)$ holds. Then, by the Dulac criteria, (1.3) admits no limit cycles or separatrix cycles. Proposition 2.3 shows that $\hat{E}=(\hat{x}, \hat{y})$ is locally asymptotically stable when $\left(\mathrm{H}_{1}\right)$ holds. On the other hand, (1.3) admits only four equilibria $E_{i}(i=0,1,2)$ and $\hat{E}$. Also, Proposition 2.1 shows that $E_{i}(i=0,1,2)$ are all unstable when $\alpha r_{2} k<a r_{1} \beta+r_{1} r_{2} c k$ holds. So, according to Proposition 3.1 and the Poincaré-Bendixson theorem, $\hat{E}$ is globally asymptotically stable.

Theorem 4.4 Suppose that $\left(\mathrm{H}_{0}\right)$ holds, further assume that

$$
\left(\mathrm{H}_{5}\right) \quad \frac{\alpha b \hat{y}}{a}+\frac{\alpha M_{2}}{2 k}+\frac{\alpha(a+b \hat{x})}{2 a}<p(a+b \hat{x}+c \hat{y}) \quad \text { and } \quad M_{2} a<k(a-b \hat{x})
$$

hold, then (1.3) has a unique positive equilibrium $\hat{E}=(\hat{x}, \hat{y})$ which is globally asymptotically stable.

Proof Let $(x(t), y(t))$ be any positive solution of (1.3). According to Proposition 2.2, (1.3) has a unique positive equilibrium $\hat{E}=(\hat{x}, \hat{y})$. From $\left(\mathrm{H}_{5}\right)$, we can choose an $\varepsilon>0$ such that

$$
\frac{\alpha b \hat{y}}{a}+\frac{\alpha\left(M_{2}+\varepsilon\right)}{2 k}+\frac{\alpha(a+b \hat{x})}{2 a}<p(a+b \hat{x}+c \hat{y}) \text { and } \quad \frac{M_{2}+\varepsilon}{2 k}+\frac{a+b \hat{x}}{2 a}<1 .
$$

Moreover, it follows from Proposition 3.1 that there exists $T>0$ such that

$$
0<y(t) \leq M_{2}+\varepsilon \quad \text { for } t \geq T
$$

Let $V(x, y)=V_{1}(x, y)+V_{2}(x, y)$, where $V_{1}(x, y)=(a+b \hat{x}+c \hat{y})\left(x-\hat{x}-\hat{x} \ln \left(\frac{x}{\hat{x}}\right)\right)$ and $V_{2}(x, y)=$ $\frac{\alpha(\hat{x}+k)}{\beta}\left(y-\hat{y}-\hat{y} \ln \left(\frac{y}{\hat{y}}\right)\right)$. Calculating the derivative of $V$ along the solution of system (1.3), we have

$$
\begin{aligned}
\dot{V}(x, y)= & (a+b \hat{x}+c \hat{y})(x-\hat{x})\left(r_{1}-p x-\frac{\alpha y}{a+b x+c y}\right)+\frac{\alpha(\hat{x}+k)}{\beta}(y-\hat{y})\left(r_{2}-\frac{\beta y}{x+k}\right) \\
= & (a+b \hat{x}+c \hat{y})(x-\hat{x})\left(-p(x-\hat{x})+\frac{\alpha \hat{y}}{a+b \hat{x}+c \hat{y}}-\frac{\alpha y}{a+b x+c y}\right) \\
& +\frac{\alpha(\hat{x}+k)}{\beta}(y-\hat{y})\left(\frac{\beta \hat{y}}{\hat{x}+k}-\frac{\beta y}{x+k}\right)
\end{aligned}
$$




$$
\begin{aligned}
& =\left(\frac{\alpha b \hat{y}}{a+b x+c y}-p(a+b \hat{x}+c \hat{y})\right)(x-\hat{x})^{2}-\alpha(y-\hat{y})^{2} \\
& +\left(\frac{\alpha y}{x+k}-\frac{\alpha(a+b \hat{x})}{a+b x+c y}\right)(x-\hat{x})(y-\hat{y}) \\
& \leq\left(\frac{\alpha b \hat{y}}{a+b x+c y}-p(a+b \hat{x}+c \hat{y})\right)(x-\hat{x})^{2}-\alpha(y-\hat{y})^{2} \\
& +\left(\frac{\alpha y}{x+k}+\frac{\alpha(a+b \hat{x})}{a+b x+c y}\right) \frac{(x-\hat{x})^{2}+(y-\hat{x})^{2}}{2} \\
& \leq\left(\frac{\alpha b \hat{y}}{a}-p(a+b \hat{x}+c \hat{y})\right)(x-\hat{x})^{2}-\alpha(y-\hat{y})^{2} \\
& +\left(\frac{\alpha y}{k}+\frac{\alpha(a+b \hat{x})}{a}\right) \frac{(x-\hat{x})^{2}+(y-\hat{x})^{2}}{2} \\
& =\left(\frac{\alpha b \hat{y}}{a}-p(a+b \hat{x}+c \hat{y})+\frac{\alpha y}{2 k}+\frac{\alpha(a+b \hat{x})}{2 a}\right)(x-\hat{x})^{2} \\
& +\left(\frac{\alpha y}{2 k}+\frac{\alpha(a+b \hat{x})}{2 a}-\alpha\right)(y-\hat{y})^{2} \\
& \leq\left(\frac{\alpha b \hat{y}}{a}-p(a+b \hat{x}+c \hat{y})+\frac{\alpha\left(M_{2}+\varepsilon\right)}{2 k}+\frac{\alpha(a+b \hat{x})}{2 a}\right)(x-\hat{x})^{2} \\
& +\alpha\left(\frac{M_{2}+\varepsilon}{2 k}+\frac{a+b \hat{x}}{2 a}-1\right)(y-\hat{y})^{2} .
\end{aligned}
$$

According to (4.13) and (4.14), $\dot{V}(x, y)<0$ strictly for all $x, y>0$ except the positive equilibrium $\hat{E}=(\hat{x}, \hat{y})$, where $\dot{V}(x, y)=0$. Thus, $V(x, y)$ satisfies Lyapunov's asymptotic stability theorem, and the positive equilibrium $\hat{E}$ of system (1.3) is globally asymptotically stable. This ends the proof of Theorem 4.4.

\section{Conclusion}

In this paper, we consider a predator-prey model with modified Leslie-Gower and Beddington-DeAngelis functional response. We discuss the structure of nonnegative equilibria and their local stability. Also, the permanence of the system is investigated. By applying the fluctuation lemma, qualitative analysis and Lyapunov direct method, respectively, three sufficient conditions on the global asymptotic stability of a positive equilibrium are obtained. Compare Theorem 4.2 with Theorem 4.3. Since $\left(\mathrm{H}_{2}\right)$ contains $\left(\mathrm{H}_{0}\right)$, what will happen when $\left(\mathrm{H}_{0}\right)$ and $\left(\mathrm{H}_{4}\right)$ hold? This is a further problem, which can be studied in the future.

Competing interests

The author declares that he has no competing interests.

Author's contributions

The author wrote the manuscript carefully, read and approved the final manuscript.

Acknowledgements

The author would like to thank the two anonymous referees for their constructive suggestions on improving the presentation of the paper. This research is supported by the Foundation of Fujian Education Bureau (JA13365).

Received: 21 October 2013 Accepted: 24 February 2014 Published: 13 March 2014

References

1. Berryman, AA: The origins and evolution of predator-prey theory. Ecology 75, 1530-1535 (1992) 
2. Leslie, $\mathrm{PH}$ : Some further notes on the use of matrices in population mathematics. Biometrika 35, $213-245$ (1948)

3. Leslie, PH: A stochastic model for studying the properties of certain biological systems by numerical methods. Biometrika 45, 16-31 (1958)

4. Korobeinikov, A: A Lyapunov function for Leslie-Gower predator-prey models. Appl. Math. Lett. 14, $697-699$ (2001)

5. Hsu, SB, Hwang, TW: Global stability for a class of predator-prey systems. SIAM J. Appl. Math. 55, 763-783 (1995)

6. Hsu, SB, Hwang, TW: Uniqueness of limit cycles for a predator-prey system of Holling and Leslie type. Can. Appl. Math. Q. 6, 91-117 (1998)

7. Hsu, SB, Hwang, TW: Hopf bifurcation analysis for a predator-prey system of Holling and Leslie type. Taiwan. J. Math. 3, 35-53 (1999)

8. Yuan, S, Song, Y: Bifurcation and stability analysis for a delayed Leslie-Gower predator-prey system. IMA J. Appl. Math. 74, 574-603 (2009)

9. May, RM: Stability and Complexity in Model Ecosystems. Princeton University Press, Princeton (2001)

10. Holling, CS: The functional response of predator to prey density and its role in mimicry and population regulation. Mem. Entomol. Soc. Can. 45, 1-60 (1965)

11. Aziz-Alaoui, MA, Daher Okiye, M: Boundedness and global stability for a predator-prey model with modified Leslie-Gower and Holling-type II schemes. Appl. Math. Lett. 16, 1069-1075 (2003)

12. Yu, SB: Global asymptotic stability of a predator-prey model with modified Leslie-Gower and Holling-type II schemes. Discrete Dyn. Nat. Soc. 2012, Article ID 208167 (2012)

13. Nindjin, AF, Aziz-Alaoui, MA, Cadivel, M: Analysis of a predator-prey model with modified Leslie-Gower and Holling-type II schemes with time delay. Nonlinear Anal., Real World Appl. 7, 1104-1118 (2006)

14. Yafia, R, El Adnani, F, Talibi Alaoui, H: Stability of limit cycle in a predator-prey model with modified Leslie-Gower and Holling-type II schemes with time delay. Appl. Math. Sci. 1, 119-131 (2007)

15. Yafia, R, El Adnani, F, Talibi Alaoui, H: Limit cycle and numerical similations for small and large delays in a predator-prey model with modified Leslie-Gower and Holling-type II schemes. Nonlinear Anal., Real World Appl. 9, 2055-2067 (2008)

16. Nindjin, AF, Aziz-Alaoui, MA: Persistence and global stability in a delayed Leslie-Gower type three species food chain. J. Math. Anal. Appl. 340, 340-357 (2008)

17. Gakkhar, S, Singh, B: Dynamics of modified Leslie-Gower-type prey-predator model with seasonally varying parameters. Chaos Solitons Fractals 27, 1239-1255 (2006)

18. Guo, HJ, Song, XY: An impulsive predator-prey system with modified Leslie-Gower and Holling type II schemes. Chaos Solitons Fractals 36, 1320-1331 (2008)

19. Song, $X Y, L i, Y F$ : Dynamic behaviors of the periodic predator-prey model with modified Leslie-Gower Holling-type II schemes and impulsive effect. Nonlinear Anal., Real World Appl. 9, 64-79 (2008)

20. Zhu, Y, Wang, K: Existence and global attractivity of positive periodic solutions for a predator-prey model with modified Leslie-Gower Holling-type II schemes. J. Math. Anal. Appl. 384, 400-408 (2011)

21. Liu, M, Wang, K: Dynamics of a Leslie-Gower Holling-type II predator-prey system with Lévy jumps. Nonlinear Anal. 85, 204-213 (2013)

22. Kar, TK, Ghorai, A: Dynamic behaviour of a delayed predator-prey model with harvesting. Appl. Math. Comput. 217, 9085-9104 (2011)

23. Huo, H, Wang, X, Chavez, C: Dynamics of a stage-structured Leslie-Gower predator-prey model. Math. Probl. Eng. 2011, Article ID 149341 (2011)

24. Li, Z, Han, M, Chen, F: Global stability of a stage-structured predator-prey model with modified Leslie-Gower and Holling-type II schemes. Int. J. Biomath. 5, 1250057 (2012)

25. Liu, C, Zhang, Q, Huang, J: Stability analysis of a harvested prey-predator model with stage structure and maturation delay. Math. Probl. Eng. 2013, Article ID 329592 (2013). doi:10.1155/2013/329592

26. Gupta, RP, Chandra, P: Bifurcation analysis of modified Leslie-Gower predator-prey model with Michaelis-Menten type prey harvesting. J. Math. Anal. Appl. 398, 278-295 (2013)

27. Ji, CY, Jiang, DQ, Shi, NZ: Analysis of a predator-prey model with modified Leslie-Gower and Holling-type II schemes with stochastic perturbation. J. Math. Anal. Appl. 359, 482-498 (2009)

28. Ji, CY, Jiang, DQ, Shi, NZ: A note on a predator-prey model with modified Leslie-Gower and Holling-type II schemes with stochastic perturbation. J. Math. Anal. Appl. 377, 435-440 (2011)

29. Lian, F, Xu, Y: Hopf bifurcation analysis of a predator-prey system with Holling type IV functional response and time delay. Appl. Math. Comput. 215, 1484-1495 (2009)

30. Arditi, R, Saiah, H: Empirical evidence of the role of heterogeneity in ratio-dependent consumption. Ecology 73 , 1544-1551 (1992)

31. Arditi, R, Ginzburg, LR, Akcakaya, HR: Variation in plankton densities among lakes: a case for ratio-dependent models. Am. Nat. 138, 1287-1296 (1991)

32. Gutierrez, AP: The physiological basis of ratio-dependent predator-prey theory: A metabolic pool model of Nicholson's blowflies as an example. Ecology 73, 1552-1563 (1992)

33. Arditi, R, Ginzburg, LR: Coupling in predator-prey dynamics: ratio-dependence. J. Theor. Biol. 139, $311-326$ (1989)

34. Arditi, R, Perrin, N, Saiah, H: Functional response and heterogeneities: an experiment test with cladocerans. Oikos 60, 69-75 (1991)

35. Yao, Z, Xie, S, Yu, N: Dynamics of cooperative predator-prey system with impulsive effects and Beddington-DeAngelis functional response. J. Egypt. Math. Soc. 21, 213-223 (2013)

36. Beretta, E, Kuang, Y: Global analysis in some delayed ratio-dependent predator-prey systems. Nonlinear Anal. 32, 381-408 (1998)

37. Liang, Z, Pan, H: Qualitative analysis of a ratio-dependent Holling-Tanner mode. J. Math. Anal. Appl. 334, 954-964 (2007)

38. Saha, T, Chakrabarti, C: Dynamical analysis of a delayed ratio-dependent Holling-Tanner predator-prey model. J. Math. Anal. Appl. 358, 389-402 (2009)

39. Liu, J, Zhang, Z, Fu, M: Stability and bifurcation in a delayed Holling-Tanner predator-prey system with ratio-dependent functional response. J. Appl. Math. 2012, Article ID 384293 (2012) 
40. Beddington, JR: Mutual interference between parasites or predators and its effect on searching efficiency. J. Anim. Ecol. 44, 331-340 (1975)

41. DeAngelis, DL, Goldstein, RA, O'Neill, RV: A model for trophic interaction. Ecology 56, 881-892 (1975)

42. Skalski, GT, Gilliam, JF: Functional responses with predator interference: viable alternatives to the Holling type II model. Ecology 82, 3083-3092 (2001)

43. Lu, Z, Liu, X: Analysis of a predator-prey model with modified Holling-Tanner functional response and time delay. Nonlinear Anal., Real World Appl. 9, 641-650 (2008)

44. Zhang, J: Bifurcation analysis of a modified Holling-Tanner predator-prey model with time delay. Appl. Math. Model. 36, 1219-1231 (2012)

45. Cantrell, R, Cosner, C: On the dynamics of predator-prey models with the Beddington-DeAngelis functional response J. Math. Anal. Appl. 257, 206-222 (2001)

46. Liu, ZH, Yuan, R: Stability and bifurcation in a delayed predator-prey system with Beddington-DeAngelis functional response. J. Math. Anal. Appl. 296, 521-537 (2004)

47. Dimitrov, DT, Kojouharov, HV: Complete mathematical analysis of predator-prey models with linear prey growth and Beddington-DeAngelis functional response. Appl. Math. Comput. 162, 523-538 (2005)

48. Lin, G, Hong, Y: Delay induced oscillation in predator-prey system with Beddington-DeAngelis functional response. Appl. Math. Comput. 190, 1296-1311 (2007)

49. Chen, WY, Wang, MX: Qualitative analysis of predator-prey models with Beddington-DeAngelis functional response and diffusion. Math. Comput. Model. 42, 31-44 (2005)

50. Xiao, HB: Positive equilibrium and its stability of the Beddington-DeAngelis's type predator-prey dynamical system. Appl. Math. J. Chin. Univ. Ser. B 21, 429-436 (2006)

51. Hwang, T: Uniqueness of limit cycles of the predator-prey system with Beddington-DeAngelis functional response. J. Math. Anal. Appl. 290, 113-122 (2004)

52. Chen, FD: On a nonlinear non-autonomous predator-prey model with diffusion and distributed delay. J. Comput. Appl. Math. 180, 33-49 (2005)

53. Hirsch, W, Hanisch, H, Gabriel, J: Differential equation models of some parasitic infection: methods for the study of asymptotic behavior. Commun. Pure Appl. Math. 38, 733-753 (1985)

54. Tineo, A: Asymptotic behaviour of positive solutions of the nonautonomous Lotka-Volterra competition equations. Differ. Integral Equ. 6, 419-457 (1993)

\section{Submit your manuscript to a SpringerOpen ${ }^{\circ}$ journal and benefit from:}

- Convenient online submission

Rigorous peer review

- Immediate publication on acceptance

- Open access: articles freely available online

- High visibility within the field

- Retaining the copyright to your article 